\title{
Band Gap Energy for SiC Thin Films Prepared By TEACO Laser Irradiated With Nuclear Radiation
}

\author{
Asia H. AI-Mashhdani * \\ Date of acceptance $20 / 7 / 2002$
}

\begin{abstract}
The effect of high energy radiation on the energy gap of compound semiconductor Silicon Carbide $(\mathrm{SiC})$ are viewed. Emphasis is placed on those effects which can be interpreted in terms of energy levels. The goal is to develop semiconductors operating at high temperature with low energy gaps by induced permanent damage in $\mathrm{SiC}$ irradiated by gamma source.

$\mathrm{TEACO}_{2}$ laser used for producing $\mathrm{SiC}$ thin films. Spectrophotometer lambda $\mathrm{UV}$, Visible instrument is used to determine energy gap $\left(\mathrm{E}_{\mathrm{g}}\right)$. Co- 60 , Cs-137, and Sr-90 are used to irradiate $\mathrm{SiC}$ samples for different time of irradiation. Possible interpretation of the changing in $\mathrm{E}_{\mathrm{g}}$ values as the time of irradiation change is discussed.
\end{abstract}

Key words: Nuclear radiation, Band gap energy, SiC thin film.

\section{Introduction}

Most who work in the field of nuclear hardening of electronic equipment are aware that many aspects of their efforts rely on related facts obtained over a long period of time. The big picture of nuclear hardening includes some scientific endeavors, yet analyses are being made that apply a kind of art. The total environment, which consists of radiation from various sources (fission, fusion, space radiation's, nuclear weapons), affects matter down to the subatomic level and is quite dependent on the domain of influence. The dominant failure mechanism in almost every design problem is lifetime degradation. It causes a decrease in power output [1].

It is necessary to obtain the functional dependence of basic semiconductor properties on radiation flounce and energy. There are two basic aspects of the interaction of radiation with semiconductor. A large fraction of the energy of an incident energetic particles or photons goes into electronic processes (excitation and ionization), and this produces a temporary or transient disturbance in the semiconductor which disappears shortly after the radiation source is removed. The remainder of the incident energy goes into atomic processes, and produces displacements of atoms within the crystal lattice. The fraction of these displacements which remain after long times 1000 second at room temperature will be called permanent damage.

The interaction of energetic electrons with semiconductor is fairly easy to analyze. If the energetic electron passes sufficiently close to the nucleus (so that coulomb screening is in effective), then a coulomb scattering event will occur. During this event the electron will transfer some of its energy

*Department of Physics, College of Science, University of Baghdad, Jadiria, Baghdad, 
to the lattice atom. The maximum amount of energy that can be transferred is given by:

$$
\mathrm{T}_{\mathrm{m}}=\frac{2\left(\varepsilon_{\mathrm{e}}+2 \mathrm{~m}_{\mathrm{e}} \mathrm{c}^{2}\right)}{M \mathrm{c}^{2}} \varepsilon_{\mathrm{e}}
$$

Where $\varepsilon_{\mathrm{e}}, \mathrm{m}_{\mathrm{e}}=$ electron's energy and mass, and $\mathrm{c}=$ velocity of light, and $\mathrm{M}$ $=$ lattice atom mass. If the energy transferred to the lattice atom is greater than a certain amount, the lattice atom will be displayed to an interstitial position. This energy is between 10 and $25 \mathrm{eV}$. The displaced atom will leave behind a vacancy in the crystal lattice. The resulting vacancyinterstitial pair is called a Frenkel defect. But the effects of ionizing radiation are other than the creation of permanent displacement type damage, the energetic radiation, create electron-hole pairs by exciting electrons across the forbidden gap. As a result, whenever gamma rays are incident on a semiconductor, there is an increase in the density of electrons and holes (due to pair generation) which will decay back to the original densities if the ionizing source is removed

In general, it is found that the average energy required to create an electron-hole pair is roughly three times the band gap of the semiconductor. This is because some of the energy is lost through other mechanism (for $\mathrm{SiC}$ semiconductor the band gap energy is $3 \mathrm{eV}$ and radiation ionization energy is $9 \mathrm{eV}$ ) [2]

In this paper, the effect of ionization radiation on the energy gap for $\mathrm{SiC}$ semiconductors are studied. One of the reasons for the initial work on $\mathrm{SiC}$ was its possible application for devices operating at high temperature due to its high energy gaps and its chemical inertness $[3,4]$. The high energy gaps of the $\mathrm{SiC}$ semiconductor, make them attractive as solid-state ultra-violet detectors, moreover, the chemical inertness of $\mathrm{SiC}$ means that high operating temperatures should be permissible so that the material could be used as a flame detector, in which application its insensitivity to longer wavelength radiation is an advantage. Detectors made from $\mathrm{SiC}$ with response peaks around $0.28 \mu \mathrm{m}[5]$.

\section{Materials and Methods}

Fig.(1) shows the process which used for producing silicon carbide thin films by using $\mathrm{TEACO}_{2}$ laser to induced reaction in the gas phase, $\mathrm{C}_{2} \mathrm{H}_{4}$ have been used as additive to $\mathrm{SiH}_{4}$ [6]. Reactant gases that vibrationally heated by absorbing energy emitted from $\mathrm{TEACO}_{2}$ laser decomposes through collision assisted multiple photon dissociation causing $\mathrm{SiC}$ thin films, their thickness is 5000 A.

The prepared samples were irradiated by different gamma doses from Cs-137 (0.662 MeV) and Co-60 (1.173 and $1.333 \mathrm{MeV}$ ) with same activity $(0.2 \mathrm{mCi})$ and beta doses $\mathrm{Sr}$ 90 with different activities (0.1 and 1.0 $\mathrm{mCi})$ respectively. The irradiation facility is at the College of Science, Baghdad University. Irradiation was carried out in air at room temperature and at an intermediate value of relative humidity $(60 \%)$.

\section{Results and Discussion}

The results of absorption and transmission data of light for any wavelength which are taken by 
Spectrophotometer type lambda- UV, VISIBLE for wavelengths $(\lambda)$ between (200-1100 nm). The measurement of the optical energy gap is done by the following: when the light with an intensity $\mathrm{I}_{\mathrm{o}}$ falls on a material with thickness $\mathrm{X}$, it obeys the following equation:

$$
I=I_{\circ} \exp (-\alpha X)
$$

Where $\mathrm{I}$ is transmitted intensity and $\alpha$ is absorption coefficient, $\mathrm{I} / \mathrm{l}_{\mathrm{o}}$ represents the transmittance $(\mathrm{T})$ and the absorb (A)

$$
\begin{aligned}
\mathrm{A} & =\log (\mathrm{l} / \mathrm{T}) \\
\mathrm{T} & =\exp (-2.303 \mathrm{~A}) \\
\alpha & =2.303(\mathrm{~A} / \mathrm{X})[\alpha \text { was }
\end{aligned}
$$

calculated at every wavelength ( $\lambda$ ) ]

The absorbance spectrum of the unirradiated substrate was taken as the reference to determine the absorption of the irradiated films. The absorption in non crystalline materials are normally analyzed in the Tauc Eq. $\left[\alpha h v=B\left(h v-E_{g}\right)\right]$, which is used to find the type of the optical transition and optical energy gap, which could be deduced from the best straight line can be drawn between $(\alpha h v)$ versus $(h v)$. Where $\mathrm{B}$ is constant $=4 \pi \sigma_{\circ}=\mathrm{nCE}_{\infty}$, $\mathrm{C}$ is the speed of light, $\sigma_{0}$ is the extrapolated d.c. conductivity at $\mathrm{T}=\infty, \mathrm{n}$ is the refractive index and $\mathrm{E}_{\infty}$ is an energy which is a measure of the extent of band tailing, normally obtained from Urbach law [7]. Table (1) presented the results of the energy gap and time of irradiation for $\mathrm{SiC}$ samples.

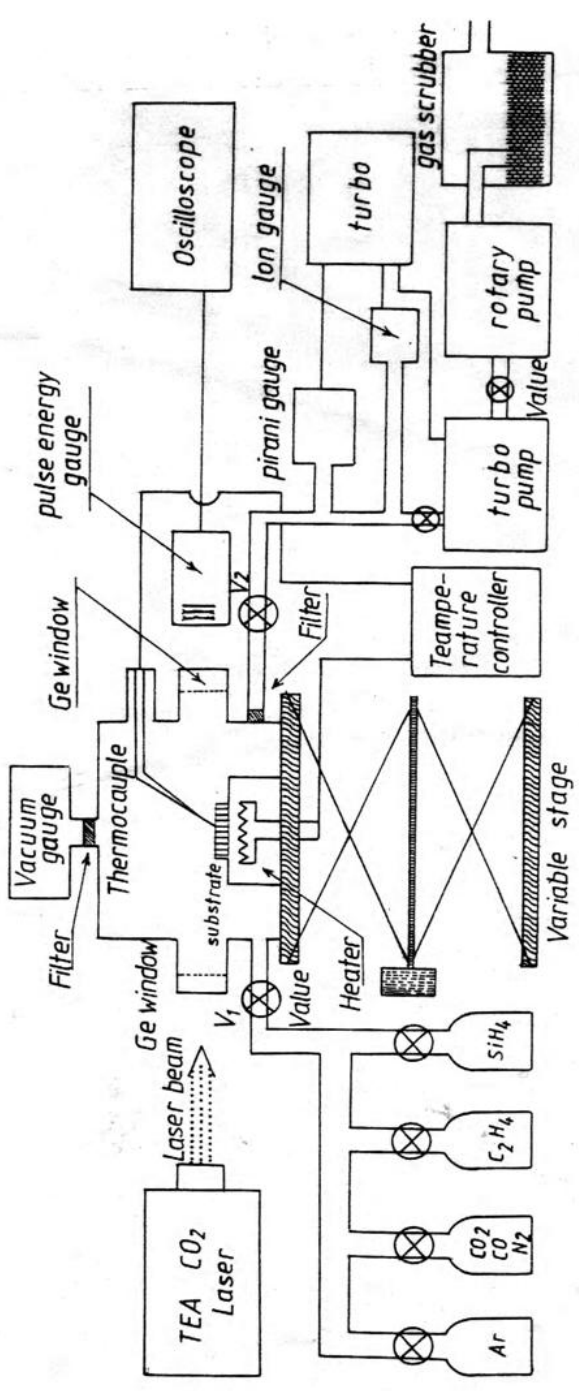

Fig.(1) Block diagram deposition system 
Table (1): Values of band gap energy for SiC samples exposed to gamma and beta rays as a function of exposure time

\begin{tabular}{|c|c|c|c|c|c|c|c|}
\hline $\begin{array}{c}\text { Time } \\
(\mathbf{m i n})\end{array}$ & $\begin{array}{c}\mathbf{E}_{\mathbf{g}} \\
\text { for sample } \\
\text { irradiated by } \\
\text { Cs-137 }\end{array}$ & $\begin{array}{c}\text { Time } \\
(\mathbf{m i n})\end{array}$ & $\begin{array}{c}\mathbf{E}_{\mathbf{g}} \\
\text { for sample } \\
\text { irradiated by } \\
\mathbf{C o - 6 0}\end{array}$ & $\begin{array}{c}\text { Time } \\
(\mathbf{m i n})\end{array}$ & $\begin{array}{c}\mathbf{E}_{\mathbf{g}} \\
\text { for sample } \\
\text { irradiated by } \\
\text { Sr-90 (0.1 mCi) }\end{array}$ & $\begin{array}{c}\text { Time } \\
(\mathbf{m i n})\end{array}$ & $\begin{array}{c}\mathbf{E}_{\mathbf{g}} \\
\text { for sample } \\
\text { irradiated by } \\
\text { Sr-90 (1 mCi) }\end{array}$ \\
\hline 0 & 1.55 & 0 & 1.50 & 0 & 1.45 & 0 & 1.55 \\
\hline 30 & 1.15 & 30 & 1.30 & 30 & 1.40 & 30 & 1.35 \\
\hline 60 & 0.85 & 60 & 1.20 & 60 & 1.35 & 60 & 1.25 \\
\hline 90 & 0.35 & 90 & 1.05 & 90 & 0.85 & 90 & 1.15 \\
\hline 120 & 0.25 & 120 & 0.70 & $* 60$ & 1.05 & $* 60$ & 1.20 \\
\hline$* 30$ & 0.65 & $* 4320$ & 0.95 & $* 120$ & 1.25 & $* 120$ & 1.35 \\
\hline$* 90$ & 0.90 & --- & & $* 180$ & 1.45 & $* 180$ & 1.55 \\
\hline$* 3600$ & 0.90 & & & & & & \\
\hline
\end{tabular}

\section{*represents to the values that the sources are removed}

The variation of $\mathrm{E}_{\mathrm{g}}$ versus time for irradiated samples is presented in Figs, (2) and (3). SiC samples revealed optical direct transitions between bands which leads to energy band gap nearly $1.50 \mathrm{eV}$ but different if these samples irradiated by gamma rays and beta particles. This difference is $=0.5 \mathrm{eV}$ for each time .Its clear from these Figs., that the values of $E_{g}$ decrease with either increasing dose rate (time of irradiation) and decreasing the energy of incident photons Fig. (2) The effect of energy photons on the $\mathrm{E}_{\mathrm{g}}$ for the irradiated samples, it shows a reduced in $E_{g}$ as the photons energy decrease, this is means that the increase in photons energy result in decreases in the probability of the interaction of photons with matter. It is known, that, below $1 \mathrm{MeV}$, photoelectric effect predominates. The late effects of the $\mathrm{SiC}$ samples irradiated by gamma photons (stored for a period of 4320 hours under atmospheric conditions at room temperature) were also detected. The effect of the ionizing radiation on the $\mathrm{SiC}$ samples is the creation of permanent displacement - type damage if the ionizing source is removed. The defects produced by atom displacement introduce energy levels into the forbidden gap of semiconductors. These energy levels can serve as recombination centers for electrons and holes. The capture of the minority carrier (i.e. holes in n- type material) is generally the rate limiting step in the recombination process, since the number of majority carriers is much larger than the number of minority carriers.

In principle, the lifetime reflects the position in the forbidden gap of the energy level of the capture site, so that measurement of the radiation dependence of the lifetime should lead to identification of the defect energy levels produced by atom displacement.

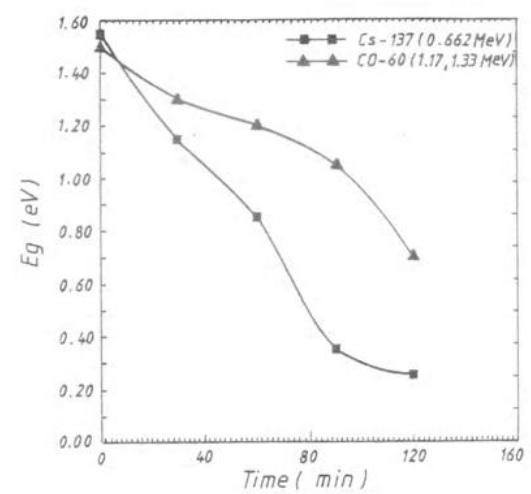

Fig. (2) The dependence of energy gap on the time of irradiation samples for different photons energy. 


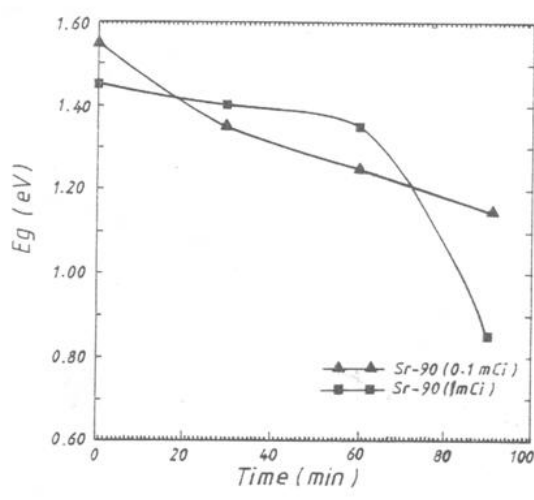

Fig. (3) The dependence of energy gap on the time of irradiation samples for different photons activities.

The beta particles create electronhole pairs by exciting electrons across the forbidden gap. As a result, whenever beta particles are incident on a semiconductor, there is an increase in the density of electrons and holes (due to pair generation) which will decay back to the original densities if the ionizing source is removed Table (1). The average energy required to create an electron - hole pair is roughly three times the band gap of the semiconductor. This is because of the energy is lost through the mechanisms. Fig. (3) shows a decrease in the energy gap values for samples irradiated by high activity beta particles ( $1 \mathrm{mCi}$ ) than samples irradiated by low activity beta source $(0.1 \mathrm{mCi})$, this behavior is attributed to increase in the probability of interaction (increase in the number of particles) with matter.

\section{Conclusions}

It may be concluded from the present investigation that low gamma doses can induce damaging effects to the $\mathrm{SiC}$ thin film and properties of their absorption. A new value of $\mathrm{E}_{\mathrm{g}}=0.9 \mathrm{eV}$ is obtained when the gamma source is removed. This new value is dependent on irradiation time, however but the $\mathrm{E}_{\mathrm{g}}$ value of $\mathrm{SiC}$ irradiated by beta particles return to initial value when the beta source is removed.

\section{References:}

1. Ricketts,L.W.,1972 ;"Fundamentals of Nuclear Hardening of Electronic Equipment", Wiley - Inter science, a Division of John Wiley and Sons, Inc., p. 175.

2. Chaffin, R. J.,1973; "Microwave Semiconductor Devices (Fundamental and Radiation Effect)", Wiley - Inter science Publication, p.137.

3. Tong, Q. Y. and Gosele, V.,1999; "Semiconductor Wafer Bonding: Science and Technology", John Wiley and Sons, Inc., p. 214.

4. Gupta,A. , Paramanik, D. Varma,S. , Jacob,C., 2004; "CVD' Growth and Characterization of SiC Thin Films", Bull. Mater. Sci., 27(5) p. 445.

5. Moss, T. S., Burrell, G. J. and Ellis, B., 1973; "Semiconductor and Opto-Electronics ", London Butterworths, p.314.

6. Al-Ethawi, A. S., AI-Azawi, H. R. and Abd Al-Amer , R., 2000; "Structural and Electrical Properties of SiC Thin Film Prepared by Laser Induced Chemical Vapor Deposition (LICVD)", Proc. of the MCE $3^{\text {rd }}$ Eng. Sci. Con., Baghdad April p.323.

7. Urbach, F., 1953; "The Long Wavelength Edge of Photo-Graphic Sensitivity and of the Electronic Absorption of Solids", Phys. Rev. 92 p. 1324.

\section{Acknowledgments}

The author wishes to thank Dr. Hamad R. Al Azawi for his keen interest and useful discussions. 


\section{تأثير الأشعة النووية على فجوة الطلقة للأغثية SiC الرقيقة المحضرة بواسطة ليزر}

\section{$\mathrm{TEACO}_{2}$}

أسبيا حمبي حدث*

*قسم الفيزياء - كلية العلوم - جامعة بغداد

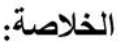

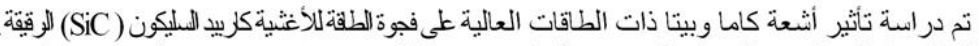

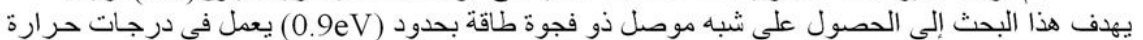

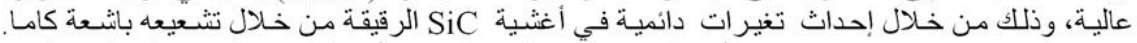
استخدم ليزر TEACO

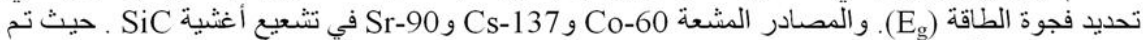

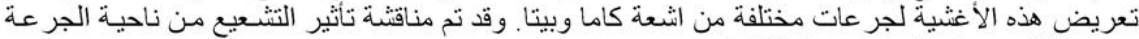

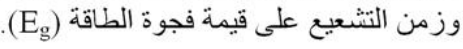

